Check for updates

Cite this: RSC Adv., 2018, 8, 29909

\title{
Cyclodextrin modified niosomes to encapsulate hydrophilic compounds
}

\author{
Noelia D. Machado, O. Fernando Silva, (D) Rita H. de Rossi \\ and Mariana A. Fernández (D)*
}

Niosomes were prepared from equimolar mixtures of two non-ionic surfactants, Span 80 and Tween 80. The capability of the vesicular systems was studied through the encapsulation of two azo dyes as molecular probes of different hydrophobicity (methyl orange (MO) and methyl yellow (MY)). To improve the efficiency of the niosomes to encapsulate the dyes, we employed an additional modification of the vesicular system, adding $\beta$-cyclodextrin $(\beta-C D)$ or a modified amphiphilic $\beta$-CD (Mod- $\beta$-CD) to the niosomes. Neither the inclusion of dyes nor the incorporation of $\beta-C D$ to the niosomes produces considerable modifications in size and morphology of the vesicles. However, in the presence of Mod- $\beta$ $C D$ the niosomes became smaller, probably due to the anchoring of the cyclodextrin at the surface of vesicles through the hydrophobic chain, altering the curvature of the outer monolayer and reducing the surface charge of the interphase. The entrapment efficiency (EE) for MY was higher than that for MO in niosomes without cyclodextrin, however, the content of $M O$ in the presence of $\beta-C D$ increased considerably. Besides, the release of this dye under the same conditions was faster and reached $70 \%$ in 24 hours whereas in the absence of the macrocycle, the release was 15\%, in the same time. UV-visible spectrophotometry and induced circular dichroism analysis allowed it to be established that MO is complexed with cyclodextrins inside vesicles, whereas MY interacts mainly with the niosome bilayer instead of with CD. Besides, the cavity of cyclodextrins is probably located in the interphase and preferably in the polar region of niosomes.

Received 11th June 2018

Accepted 13th August 2018

DOI: $10.1039 / c 8 r a 05021 j$

rsc.li/rsc-advances desired effect over an extended period of time. ${ }^{10-12}$ These systems consist of lamellar structures formed by amphiphilic molecules surrounded by an aqueous compartment. They are useful for the release of both hydrophilic and hydrophobic compounds, which are encapsulated in their internal hydrophilic compartment or in the outer lipid layer, respectively.

Vesicular systems can be of different types depending on the main components that are used in their preparation. ${ }^{13,14}$ Liposomes are the best known and they have been widely investigated as models to study the cell membrane, and as carriers for protection and/or delivery of bioactive agents. They are formed by natural or synthetic phospholipids..$^{15}$ The high costs of their formulation and low chemical stability are their main disadvantages that promote the seeking of new vesicular systems with improved properties. ${ }^{13}$

Niosomes are microscopic vesicles formed by non-ionic surfactants which form closed bilayer structures due to their amphiphilic nature. ${ }^{16,17}$ The lipophilic groups are located within the membrane while the hydrophilic groups are exposed to the aqueous medium ${ }^{18,19}$ as in liposomes. Non-ionic surfactants are preferred in comparison with ionic ones due to their ability to increase the solubility of highly water insoluble compounds, ${ }^{20}$ great compatibility with biological systems (lower toxicity, less irritation effect and non-immunogenicity), ${ }^{21}$ improved chemical
Instituto de Investigaciones en Físico-Química de Córdoba (INFIQC-CONICET), Departamento de Química Orgánica, Facultad de Ciencias Quimicas, Universidad Nacional de Córdoba, Ciudad Universitaria, Х5000HUA, Córdoba, Argentina. E-mail: marfer@fcq.unc.edu.ar 
stability against oxidation, and feasibility of surface modification. In addition, they are generally derived from renewable sources, allowing their sustainable production, ${ }^{22}$ and also are biodegradable. Niosomes allow to entrap both hydrophilic and lipophilic compounds. A lipophilic one, could interact with alkyl chains of non-ionic surfactants in the domain of the bilayers, whereas an hydrophilic compound could interact either with non-ionic surfactant polar head groups (adsorbing in the bilayers surfaces) or be located in the aqueous inner of niosomes. ${ }^{23,24}$ Due to this characteristics, niosomes present a wide range of potentials applications in cosmetics, ${ }^{25}$ pharmaceutics, ${ }^{26-28}$ food, ${ }^{29}$ and agrochemical industries.

Incorporation of some macrocycles like cyclodextrins to vesicular systems has been used as additional strategy to improve the loaded amount, stability and bioavailability of very hydrophobic compounds in liposomes. ${ }^{30-32}$ Cyclodextrins (CDs) have been employed due to their ability to form inclusion complexes with a wide range of compounds, ${ }^{33}$ however the strategies to load cyclodextrin-drug complexes in niosomes was barely used due to the need to previously form the inclusion complex before the incorporation in vesicles. ${ }^{34,35}$

In this work we prepared niosomes from equimolar mixtures of two non-ionic surfactants, Span 80 (sorbitan monooleate) and Tween 80 (polyoxyethylen (20) sorbitan monooleate), whose chemical structures are shown in Scheme 1. To evaluate the transport properties of the systems we studied the encapsulation of two azo dyes as molecular probes of different hydrophobicity (methyl orange (MO) and methyl yellow (MY)), Scheme 1. MO has a high water solubility at $20{ }^{\circ} \mathrm{C}, 5000 \mathrm{mg} \mathrm{L}{ }^{-1},{ }^{36}$ thus $\mathrm{MO}$ is a model of hydrophilic active agent. In the other way, the aqueous solubility of MY at $20^{\circ} \mathrm{C}$ is $13.6 \mathrm{mg} \mathrm{L}^{-1},{ }^{37}$ so this compound is a model of an hydrophobic active molecule.

With the aim to improve the efficiency of the niosomes as vectors of active agents, we made a modification of the vesicular system, adding $\beta$-cyclodextrin ( $\beta$-CD) to the vesicles. Additionally,
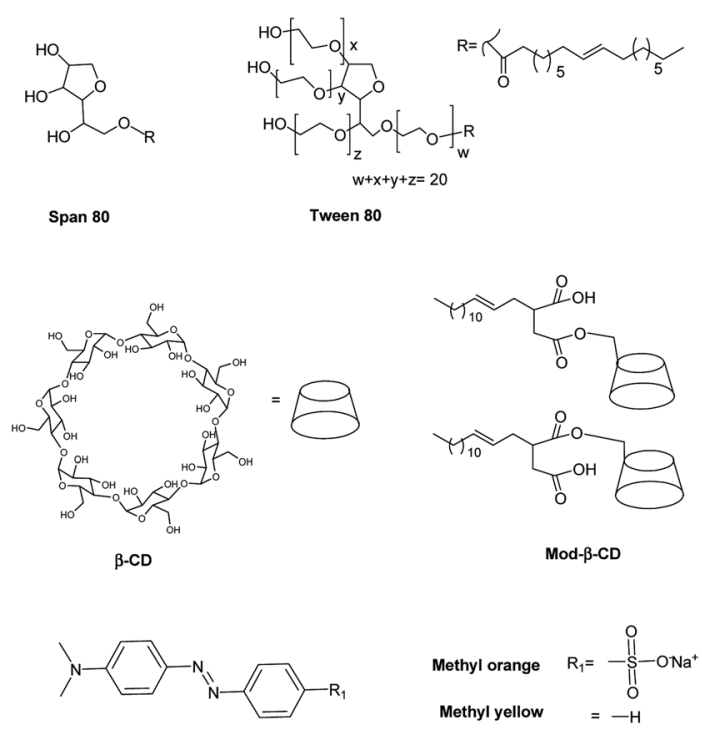

Scheme 1 Chemical structures of different molecules employed in the study. we included an amphiphilic modified $\beta$-CD in the niosomes (Mod- $\beta-C D$, Scheme 1) with the idea that this compound probably could be located in a different place than the native $\mathrm{CD}$, due to the presence of the aliphatic chain. Thus, the interaction of the active compounds with the CDs loaded niosomes could be different. Mod- $\beta$-CD has been synthetized and studied in our laboratory ${ }^{38,39}$ and it was demonstrated that it can form aggregates in aqueous and organic media, and in both systems, the cavity is available to interact with external guests. ${ }^{40-42}$

It is worth to mention that all the molecules used in this work for the synthesis of the carriers are fatty acids and sugar derivated. They are biodegradable, food grade, and their production is sustainable. The results obtained from the studies of these systems (niosomes-macrocycles) are important for further applications of these systems as delivery agents.

\section{Experimental}

\section{Materials and methods}

Materials. Span 80 (S80) was purchased to Fluka and Tween 80 (T80) to Riedel-de Haën. Methyl yellow (MY) and Methyl orange (MO) used as molecular probes were provided by Merck. $\beta$-Cyclodextrin ( $\beta$-CD) was Sigma Aldrich. Mod- $\beta$-CD was synthetized in the laboratory as was previously described. ${ }^{\mathbf{3 8 , 3 9}}$ Chloroform and methanol (HPLC quality) were obtained by J. T. Baker. Milli Q water was used in all experiments.

Preparation of niosomes. Niosomes were prepared using the thin film hydration method. Briefly, adequate quantities of nonionic surfactants (T80 and S80) were weighed in order to obtain systems with hydrophilic-lipophilic balance (HLB) at a value HLB $=10$, achieved at equimolar ratio of both surfactants. The mixtures were dissolved in chloroform in a round bottom flask and the solvent was removed under reduced pressure in a Büchi R-200 equipment, until a thin film was formed on the flask walls. The hydration step was performed using $10 \mathrm{~mL}$ of water and the suspension was shaking at $116 \mathrm{spm}$ during $30 \mathrm{~min}$ at $60{ }^{\circ} \mathrm{C}$ in a shaker water bath OLS200 Grant. The dispersion was left to equilibrate at room temperature overnight and small unilamellar vesicles (SUV) were prepared by sonication in an ultrasonic bath (Denimed of $42000 \mathrm{~Hz}$ y $170 \mathrm{~W}$ ) for $30 \mathrm{~min}$ at $60{ }^{\circ} \mathrm{C}$, and then were extruded 15 times to produce $100 \mathrm{~nm}$ diameter niosomes, using an Avanti Mini Extruder (Avanti Polar Lipids, Inc). The final surfactant concentration in the niosomes was $0.01 \mathrm{M}$.

Molecular probe loaded niosomes. For preparing MY loaded niosomes, an accurate amount of solid MY was added during the dissolution of the surfactants with chloroform. To prepare MO loaded niosomes, an aqueous solution of MO was used instead water during the hydration step, and the procedure keep unchanged during the preparation of niosomes. The purification of niosomes was performed using the dialysis method. Briefly, $3 \mathrm{~mL}$ of loaded niosomes suspension were dropped into a benzoylated dialysis tubing (Sigma Aldrich, cut off two kDa MW) and immersed in $200 \mathrm{~mL}$ of water with stirring, changing the medium for fresh water after $6 \mathrm{~h}$.

Cyclodextrins based niosomes. To incorporate the cyclodextrins in the niosomal system the procedure was slightly modified taking into consideration the different solubilities of 
cyclodextrins in water. For $\beta-\mathrm{CD}$, an aqueous solution of the macrocycle $1.5 \times 10^{-3} \mathrm{M}$ was used during the step of hydration instead water, and the remaining procedure being unchanged. To incorporate Mod- $\beta$-CD the process was different due to the low solubility of Mod- $\beta$-CD in water $\left(1 \times 10^{-4} \mathrm{M}\right)$. The procedure consisted in adding a solution of niosome $(0.01 \mathrm{M})$ to a weighted amount of solid Mod- $\beta$-CD. After that, the dispersion was shaked at $116 \mathrm{spm}$ during $24 \mathrm{~h}$ at $37^{\circ} \mathrm{C}$ to obtain a complete incorporation of the Mod- $\beta-\mathrm{CD}$ into the niosome $($ Mod- $\beta-\mathrm{CD}=$ $1.7 \mathrm{mM}$ ). For preparing niosomes with cyclodextrins loaded with MY, the thin film formed was hydrated with water or with an aqueous $\beta$-CD solution. The first system was used to dissolve Mod- $\beta$-CD. For preparing cyclodextrin niosomes with MO, the thin film was hydrated with a MO or MO: $\beta-\mathrm{CD}$ aqueous solution. The first preparation was used later to dissolve Mod- $\beta-C D$.

\section{Size and morphology determinations}

Dynamic light scattering (DLS) experiments. Size and polydispersity index (PDI) were determined using DLS analysis. The equipment was a particle analyzer Delsa ${ }^{\mathrm{TM}}$ NanoC Beckman Coulter Inc. The measurements were carried out with $658 \mathrm{~nm}$ laser at $25{ }^{\circ} \mathrm{C}$. The niosomal suspension was previously filtered through $0.45 \mu \mathrm{m}$ pore size nylon membrane filter. Standard deviations $( \pm$ S.D.) were obtained for the average value of three diameter measurements.

Transmission electron microscopy (TEM) experiments. Size and morphology of the vesicles were determined using TEM analysis. The equipment was a Jeol 1200 EX II microscope operating at an accelerating voltage of $80 \mathrm{kV}$. A drop of niosomal suspension was placed on a carbon coated copper grid and a solution of $2 \%(\mathrm{v} / \mathrm{v})$ uranyl acetate was used as staining agent.

Determination of entrapment efficiency and in vitro release studies. Entrapment Efficiency (EE) was defined as the molar ratio between the amount of molecular probe entrapped into niosomes after dialysis ([probe molecule $\left.]_{\text {entrapped }}\right)$ and the total amount of the molecule present in the non-dialyzed sample ([probe molecule $]_{\text {initial }}$ ) as it is shown in eqn (1). ${ }^{21}$ The concentration of initial and entrapped molecular probe were determined from absorption spectra of the molecular probe after the niosomal bilayer rupture with methanol. The MO and MY maximum absorbance $\lambda$ in methanol, in the presence of niosomes, were $425 \mathrm{~nm}$ and $410 \mathrm{~nm}$, respectively.

$$
\mathrm{EE}=\frac{[\text { probe molecule }]_{\text {entrapped }}}{[\text { probe molecule }]_{\text {initial }}} \times 100
$$

Release studies were performed using Franz diffusion cells. The experiments were carried out at $37{ }^{\circ} \mathrm{C}$ under stirring, and with a dialysis benzoylated membrane (Sigma Aldrich cut off 2 kDa MW) separating both compartments. The donor compartment was filled with molecular probe loaded niosomes and the receptor compartment was filled with water.

At predetermined time intervals and up to $24 \mathrm{~h}$, receptor solution were sampled for analysis and replaced with the same volume of fresh solution. The receptor compartment volume was constant during all the experiment. This procedure was needed to ensure sink and quantitative conditions ${ }^{43}$ to determine small amounts of permeated molecular probe. The dye content in samples was measured by UV-visible spectrophotometry using $4 \mathrm{~cm}$ path length quartz cells. The permeation of molecular probe aqueous solution was also investigated in the same way, and used as control.

Spectrophotometric studies. The absorption spectra of cyclodextrins in niosomes using molecular probes were measured in a Shimadzu 1800 spectrophotometer.

Induced circular dichroism (ICD) experiments were carried out using a JASCO J-810 instrument. The path length used in all spectroscopic experiments was $1 \mathrm{~cm}$ unless otherwise mentioned.

\section{Results and discussion}

Niosomes were prepared from a mixture of non-ionic surfactants, whose chemical structures are shown in Scheme 1. A practical, adimensional and empirical parameter that allows predicting vesicles formation is called hydrophilic-lipophilic balance (HLB), which is a measure of the relative contribution of the hydrophilic and lipophilic regions of the surfactant molecules. It was reported in literature that compounds or blends with values of HLB $>12$ are water soluble and tend to form micelles, although in some cases, with the adding of some additives as cholesterol, can form vesicles. On the other way, compounds with HLB $<6$ are soluble in organic solvents and have the tendency to form water in oil (W/O) emulsions. Instead, compounds with an intermediate value of HLB in the range of $7-11$, can form bilayers and in consequence vesicles..$^{13,19,21,44,45}$

In this regard, an aqueous solution of T80 ( $\mathrm{HLB}=15)$ tends to form micelles, whereas a solution of S80 $(\mathrm{HLB}=4)$ tends to form W/O emulsions as were experimentally corroborated. Each of surfactants can not form vesicles, however, an equimolar mixture of T80-S80 form vesicles as the parameter HLB $=10$ predicted. The total concentration of surfactants was $0.01 \mathrm{M}$. In the case of niosomes prepared with added $\mathrm{CD}$, the final concentration of $\beta-\mathrm{CD}$ was $1.5 \mathrm{mM}$ and of Mod- $\beta-\mathrm{CD}$ was $1.7 \mathrm{mM}$. It is worth to notice that it is not possible to dissolve this amount of Mod- $\beta$-CD in pure water.

\section{Size and morphology}

Table 1 shows the mean diameter and PDI (polydispersity index) obtained by DLS for empty and loaded niosomes in water. In general, mean diameters obtained for the different niosomal

Table 1 Mean diameter $(\mathrm{nm})$ of the T80-S80 niosomes ${ }^{a}$

\begin{tabular}{llcl}
\hline & Without CD & With $\beta-\mathrm{CD}^{a}$ & With Mod- $\beta-\mathrm{CD}^{b}$ \\
\hline MY & $110 \pm 10(0.5)$ & $106.9 \pm 0.7(0.24)$ & $72 \pm 1(0.25)$ \\
MO & $110 \pm 10(0.5)$ & $107 \pm 2(0.15)$ & $71 \pm 2(0.27)$ \\
Empty & $130 \pm 20(0.40)$ & $114.2 \pm 0.8(0.18)$ & $83 \pm 8(0.4)$
\end{tabular}

${ }^{a}$ The errors of diameters correspond to the standard deviation of three measurements. ${ }^{b}$ The values in brackets are the averaged PDI values $(n=3)$. 
formulations (with and without $\beta$-CD) were not remarkable different and had a mean diameter of $\sim 110 \mathrm{~nm}$. In addition, mean diameter does not change considerably due the entrapped molecular probe. In regards to niosomes loaded with Mod- $\beta-C D$, the size of the vesicles tends to decrease by the presence of the amphiphilic cyclodextrin, and this result suggests that this CD interacts with the vesicular aggregates. It has been demonstrated in literature that the number of carbohydrate residues in the oligosaccharide chain of the polar headgroup plays a crucial rol to determine the characteristics of the self-assembled structure such as the radius of curvature. ${ }^{46}$ In this way, it has been reported that the loading of synthetic polymer-lipids ${ }^{47}$ or hydroxypropyl- $\beta$ cyclodextrin $^{48}$ (HP- $\left.\beta-\mathrm{CD}\right)$ to liposomes leads to decrease the size of the vesicles. The authors explained that the use of polymer-lipids molecules leads to an asymmetric distribution of synthetic lipids between the outer and inner monolayers as a consequence of a significant bending of the outer layer. In case of using HP- $\beta-C D$, the cyclodextrins act shelling the surface charge of the interphase. ${ }^{48}$ However, the mechanism of particle size reduction by cyclodextrins is unclear and in literature to date are reflected the difficulties to demonstrate, in a direct way, structural evidence that show the location of CDs in surfactants assemblies. ${ }^{49}$ With regards to the mentioned above, we explained our results considering that the modified cyclodextrin could anchor at the surface of niosomes penetrating in the layer through the hydrophobic chain ${ }^{50}$ and due the insertion in the outer layer and the presence of cyclodextrin moiety, the area of polar headgroup at the interphase increase and alters the radius of curvature, and thus a decreasing in the size of niosomes was observed. Table 1 also shows that PDI values obtained for all niosomes were equal or lower than 0.5 , which indicate that the vesicles are monodisperse. ${ }^{25,29,51,52}$

Fig. 1 shows TEM images of empty niosomes (Fig. 1 A), loaded niosomes with molecular probes (Fig. $1 \mathrm{~B}$ and $\mathrm{C}$ ), and niosomes with the incorporation of $\beta$-CD (Fig. 1 D) or Mod- $\beta$-CD (Fig. $1 \mathrm{E}$ ). Fig. $1 \mathrm{~F}$ and $\mathrm{G}$ present $\beta$-CD loaded niosomes with the incorporation of MO and MY respectively.

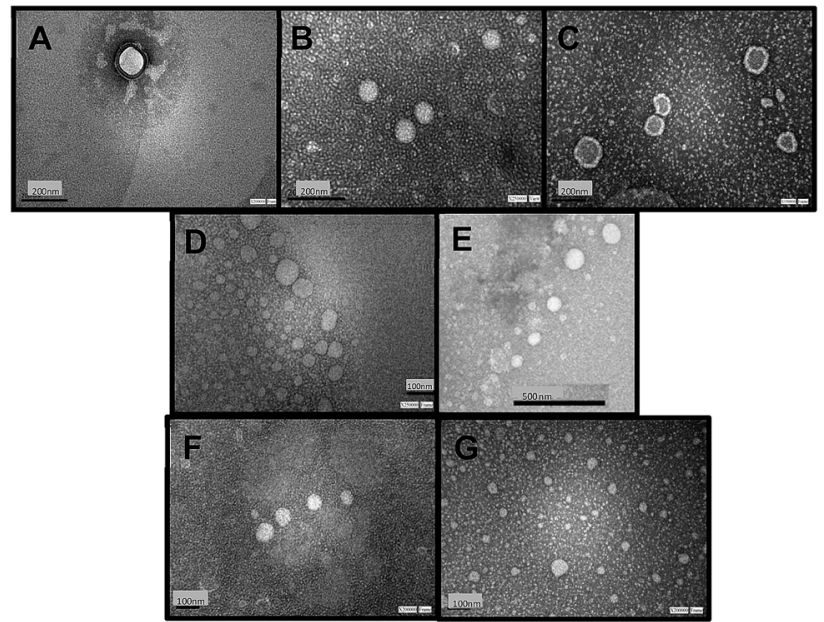

Fig. 1 TEM microphotographs of T80-S80 niosomes: (A) empty; (B) MO loaded; (C) MY loaded; (D) and (E) with $\beta-C D$ and Mod- $\beta-C D$ without molecular probes; (F) with $\beta-C D$ and $M O$; $(G)$ with $\beta-C D$ and MY. In all cases, negative stain with $2 \%(\mathrm{v} / \mathrm{v})$ uranyl acetate was employed.
The presence of molecular probes (MY or MO) do not alter the morphology of niosomes, neither empty or cyclodextrin loaded niosomes (Fig. 1). It follows that loading process and addition of the macrocycle do not modify niosomes morphology. ${ }^{24,25}$ Besides, the size of the vesicles determined from TEM measurements are in good agreement with those determined by DLS measurement.

All niosomal formulations were stable for at least 2 months storaged at $4{ }^{\circ} \mathrm{C}$. The stability of niosomes was assessed by measuring their vesicle size and morphology several times during two months.

\section{Spectrophotometric characterization of the systems}

Spectrophotometric studies were carried out using MO and MY as molecular probes with the aim to determine the location of the cyclodextrins into the vesicles.

In Fig. $2 \mathrm{~A}$, the absorption spectra of $\mathrm{MO}$ in water, $\beta$-CD aqueous solution, niosomes, and niosomes with $\beta$-CD or Mod$\beta$-CD are shown. The wavelength of MO maximum absorption
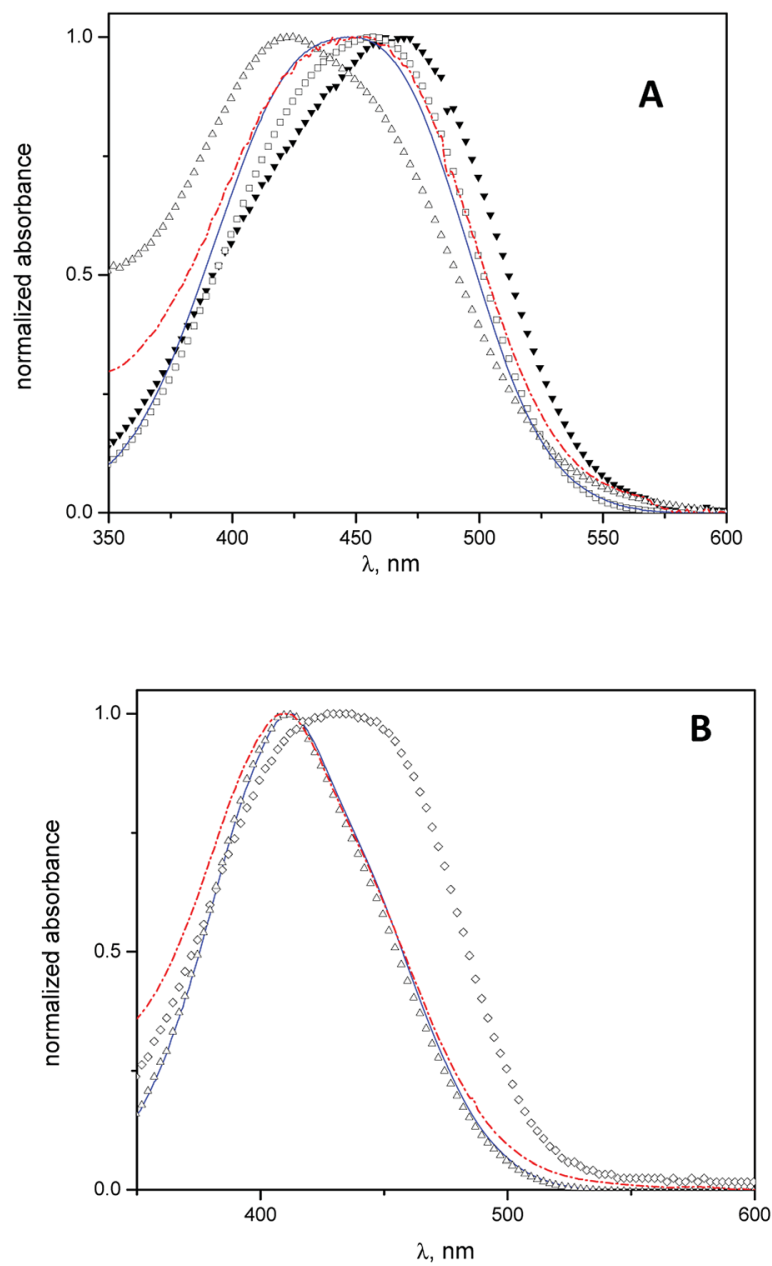

Fig. 2 Normalized UV-visible spectra of azo dyes in water (closed triangles), T80-S80 niosomes (open triangles), $\beta-C D-T 80-S 80$ niosomes (blue solid line), Mod- $\beta$-CD-T80-S80 niosomes (red dash line), and $\beta-C D$ aqueous solution (open squares). (A) MO. (B) MY. [Molecular probe $]=5 \times 10^{-5} \mathrm{M},[\beta-C D]=1.5 \times 10^{-3} \mathrm{M},[\mathrm{Mod}-\beta-C D]=1.7 \times$ $10^{-3} \mathrm{M}$, [Surf $]_{\text {total }}=0.01 \mathrm{M}$. 
shifts from $467 \mathrm{~nm}$ in water to lower wavelength $(\lambda=425 \mathrm{~nm})$ in niosomes media. This hypsochromic shift indicates that in niosomes, MO is sensing a less polar environment ${ }^{41}$ and suggest that it resides in the polar region of interphase but we cannot discard the presence of MO in the entrapped aqueous.

In presence of CDs, the results show that the MO absorption band shifts to lower wavelengths, i.e., $\lambda_{\max }=457 \mathrm{~nm}$ in $\beta-\mathrm{CD}$ aqueous solution and $\lambda_{\max }=445 \mathrm{~nm}$ in niosomes with $\beta$-CD (or Mod- $\beta-C D)$, in comparison with that obtained in water. These features were attributed to the interaction between MO and CDs by the formation of an inclusion complex. ${ }^{41}$

On the other hand, the electronic band of the complex MO:CDs in niosomes is quite different, in position and in shape, when they were compared with the spectrum of the complex MO: $\beta-C D$ in aqueous solution. We interpret our results considering that in niosomes systems, the inclusion process occurs preferably in lower polarity regions such as the interphases. We described previously that a maximum absorption wavelength of $445 \mathrm{~nm}$ corresponded to an absorption band of the inclusion complex MO:HP- $\beta-C D$ in a media with low polarity (reverse micelles of 1,4-bis(2-ethylhexyl)-sulfosuccinate, AOT). In that case we explained the results considering that the complex was formed in the confined water pool inside the reverse micelles. ${ }^{41}$

Equivalent spectrophotometric analysis was performed for the interaction of MY with niosomes, $\beta$-CD niosomes and Mod$\beta$-CD niosomes (Fig. 2B). It is shown that the maximum absorption wavelengths of $\mathrm{MY}$ are $432 \mathrm{~nm}$ in $\beta-\mathrm{CD}$ aqueous solution, and $411 \mathrm{~nm}$ in niosomes (empty or with CDs). As can be seen, there is no differences between the maximum of MY in the last three systems. These results indicate that MY interacts mainly with the niosomes bilayer, instead of with CD.

In Fig. 3 we show induced circular dichroism (ICD) spectra of MO in niosomes loaded with $\beta$-CD and niosomes loaded with Mod- $\beta$-CD. The presence of signals indicate that MO is interacting with the chiral cavity of the CDs in the niosomes. It has been fully demonstrated that when an achiral chromophore is in a chiral environment like that produced by cyclodextrin, ICD signals at wavelengths close to the maximum of absorption of the achiral compound are observed. ${ }^{53}$

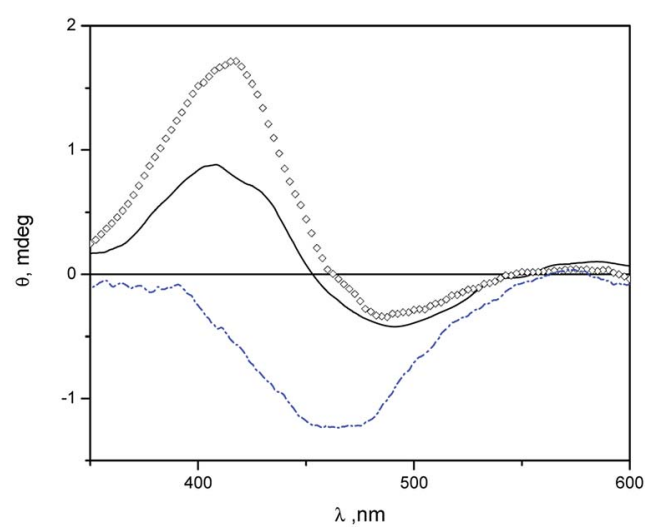

Fig. 3 ICD spectra of $M O$ in: $\beta-C D$ aqueous solution (open squares), $\beta-C D-T 80-S 80$ niosomes (solid line), and Mod- $\beta$-CD-T80-S80 (dash line) niosomes. $[\mathrm{MO}]=5 \times 10^{-5} \mathrm{M},[\beta-\mathrm{CD}]=1.5 \times 10^{-3} \mathrm{M}$, [Mod- $\beta$ $\mathrm{CD}]=1.7 \times 10^{-3} \mathrm{M}$, [Surf $]_{\text {total }}=0.01 \mathrm{M}$.
The ICD spectra of MO in aqueous solution of $\beta$-CD and in $\beta$ CD-niosomes have a splitted signal (a positive and a negative band) whereas in the system Mod- $\beta$-CD-niosomes the ICD signal of MO is negative (Fig. 3). These results indicate that the complex MO:Mod- $\beta$-CD in niosomes is different from that formed between MO: $\beta$-CD (with or without niosomes).

An empirical rule for the analysis of the inclusion complexes of cyclodextrins with chromophoric molecules states that the sign of the induced circular dichroism depends on the orientation of the transition dipole moment in the cyclodextrin's cavity. ${ }^{53-55}$ Briefly, an ICD positive signal is observed when the electric transition dipole moment of a chromophore included into the cavity is parallel to the principal axis of the cyclodextrin, and the ICD signal is negative if the electric transition dipole moment is perpendicular to the principal axis of the host. The opposite was observed with the electric transition dipole moment of a chromophore that was not included into the cavity of cyclodextrins, but it is close enough to detect the chiral effect of the macrocycle. We proposed that in the niosomes containing $\beta$-CD, MO can be included in a deeper form into the cavity, meanwhile in Mod- $\beta$-CD niosomes, due to the $\mathrm{CD}$ is anchored onto the surface of niosomes, the chromophore can be partially located into the cavity. The presence of a small negative ICD signal, besides the positive one when MO is in presence of $\beta-C D$, could be indicating that the chromophore can take different orientations in this macrocycle, probably because the cavity is more available. Additionally, no ICD signals were observed with MY:cyclodextrin niosomes. Probably due to the low water solubility and lower association constant of MY with $\beta-\mathrm{CD}$, the dye is located in the hydrophobic region of vesicles. We explained these results considering the affinities of MO for the interphase and the cavities of cyclodextrins. The association constant $\left(K_{\mathrm{as}}\right)$ for $\mathrm{MO}: \beta-\mathrm{CD}(1: 1)$ inclusion complex is $2.97 \times$ $10^{3} \mathrm{M}^{-1} \cdot{ }^{56}$ In the same conditions MY is very insoluble in water even in the presence of cyclodextrin. In literature, there is a value reported for the apparent formation constant of MY: $\beta$ CD complex at pH 1.1 of $(49 \pm 1) \times 10^{1} \mathrm{M}^{-1} \cdot{ }^{57}$ In those experimental conditions the protonated azo compound is more soluble than at pH 7, however the reported value of $K_{\text {as }}$ allow us estimate that the concentration of complex MY : $\beta$-CD $(1: 1)$ is considerably lower than the value for the complex MO : $\beta$-CD (1:1). Considering the $\log P$ values for $\mathrm{MY}^{58}$ and $\mathrm{MO},{ }^{59}$ that are 4.60 and 2.06 respectively, it is reasonable that MY shows stronger affinity for the hydrophobic region of bilayers than MO.

Considering the difference in cyclodextrins' hydrophilicity, we suggest that $\beta$-CD is probably located in the polar region of the interphase, in the outer and in the inner monolayers. The fact that $\beta-\mathrm{CD}$ can be incorporated into the polar region may be attributed to the interaction of the $\mathrm{OH}$ groups with the polar headgroup of the surfactants. It is known that the $\mathrm{OH}$ groups on the exterior of cyclodextrins can form hydrogen bonds with water, or in the same way, interact with the headgroup of surfactants. ${ }^{\mathbf{4 2 , 6 0}}$ We also know from the absorption spectra of MO that this compound is at the interphase, located close to a chiral environment, and this is another evidence of the presence of $\mathrm{CD}$ in this region of the vesicle. In the other way, the 
location of Mod- $\beta$-CD in T80-S80 niosomes was explained considering that the presence of the long hydrocarbon tail in its structure helps the incorporation into the interphase since Mod- $\beta$-CD is less soluble in water. According with the results of DLS and TEM, Mod- $\beta-C D$ is distributed mostly in the outer monolayers and thus conduct to a reduction in the size of vesicles.

The ICD experiments showed that the cavities of Mod- $\beta-C D$ are available to interact with $\mathrm{MO}$ and the negative signal indicate that the MO cannot be located in a deeper form, and we suggest that is due to the presence of the tails of surfactants that also competes for the cavities. Scheme 2 summarizes the above discussion.

\section{Entrapment efficiency (EE) and in vitro release studies}

According to eqn (1), EE was determined for niosomal formulations with and without CDs. The concentrations of the initial amount and the finally entrapped concentration of molecular probes into niosomes were determined with the corresponding calibration plot. Table 2 shows the obtained values.

It was observed that EE obtained for MY was higher than that obtained for MO in niosomes without CDs, probably due to the MY poor water solubility, therefore it has more affinity for the lipophilic bilayer of the niosome.

EE for MY was not modified by the presence of CDs, meanwhile, the value of EE for MO was slightly increased in niosomes containing $\beta-\mathrm{CD}$, however the values of $\mathrm{EE}$ in Mod- $\beta-\mathrm{CD}$ were not different from those obtained in T80-S80 niosomes (initial concentrations of the dyes were keeping constant).

We explained these results considering the affinities of MO for the interphase and the cavities of cyclodextrins. The increase in $\mathrm{EE}$ for MO due to niosomes with $\beta$-CD can be attributed to the presence of a complex between MO: $\beta$-CD since the association constant for the inclusion complex MO : $\beta$-CD $(1: 1)$ is considerable, about $3000 \mathrm{M}^{-1}$ (ref. 56) as was mentioned. In the same conditions MY is very insoluble in water even in the presence of cyclodextrin, so the amount of complex formed with the last compound is considerably lower than with MO. There are some

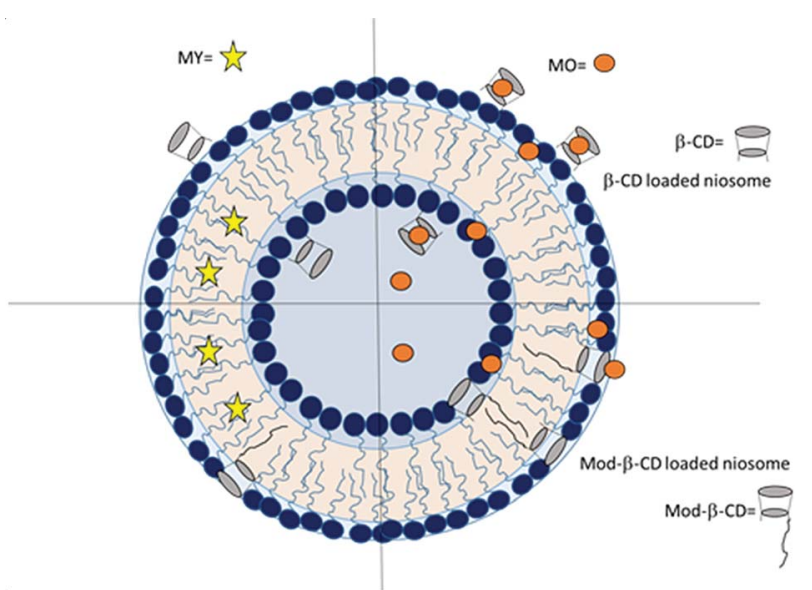

Scheme 2 Representation of the estimated different locations for CDs in T80-S80 niosomes.
Table 2 EE (\%) obtained for MY and MO loaded niosomes in water ${ }^{a}$

\begin{tabular}{lll}
\hline $\begin{array}{l}\text { Niosomes } \\
\text { formulation }\end{array}$ & MY & MO \\
\hline T80-S80 & $54 \pm 8$ & $45 \pm 8$ \\
Mod- $\beta$-CD-T80-S80 & $48 \pm 5$ & $40 \pm 5$ \\
$\beta$-CD-T80-S80 & $60 \pm 10$ & $57 \pm 9$
\end{tabular}

${ }^{a}$ The errors correspond to the standard deviation of three measurements.

reports of improvements of EE when the drug is incorporated in liposomes as drug/CD inclusion complexes in a similar way to the results here obtained. For instance, the entrapment of curcumin, a broad-spectrum anticancer drug, increased from $30 \%$ in $\mathrm{CD}$-free liposomes to $50 \%$ when hydroxypropyl- $\beta$-CD (HP- $\beta$-CD) was added to the system. ${ }^{61}$ In another study, indomethacin (a nonsteroid anti-inflammatory drug) was included in liposomes of soybean phosphatidylcholine as a complex with $\beta$-CD or HP- $\beta$-CD, obtaining improvements in the loaded amount of indomethacin of approximately 1.5 times in both cases, in comparison with the experiments without $\mathrm{CD}{ }^{62}$

In the other hand, Mod- $\beta$-CD is located among the tails of surfactants and the hydrocarbon chains are included into the cavity of CD decreasing the complex MO:Mod- $\beta$-CD. It worth to mention that not all the cavities are occupied by the tails of surfactants and many of them are able to interact with external guests as we observed from ICD measurement. The higher affinity of MO by $\beta$-CD could increase the encapsulated amount of this molecule that is included in the niosome.

In vitro release experiments were carried out in water at $37{ }^{\circ} \mathrm{C}$, and release profiles are shown in Fig. 4 .

With the aim to evaluate CDs effect in the niosomes, the release profiles were shown for comparison. It was observed that MO cumulative release from $\beta$-CD niosomes is higher than MO released from T80-S80 niosomes during elapsed time measurements. After $24 \mathrm{~h}, 70 \%$ of $\mathrm{MO}$ was released from the

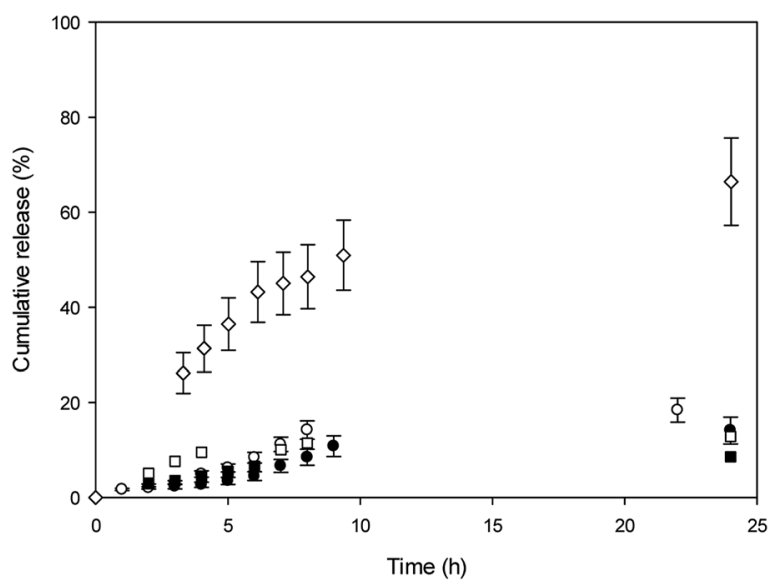

Fig. 4 Cumulative release (\%) versus time in water at $37^{\circ} \mathrm{C}$. (०) MY and $(O)$ MO from T80-S80 niosomes; $(\diamond)$ MO from $\beta$-CD-T80-S80 niosomes. ( $\square$ ) MO and (ם) MY from Mod- $\beta$-CD-T80-S80 niosomes. $[\beta-C D]=1.5 \times 10^{-3} \mathrm{M},[$ Mod- $\beta-C D]=1.7 \times 10^{-3} \mathrm{M}$, [Surf $]_{\text {total }}=$ $0.01 \mathrm{M}$. 
niosomes with $\beta-\mathrm{CD}$, whereas almost only $20 \%$ of MO was released from niosomes without the macrocycle. The initial concentration of this dye was the same in both niosome systems. We explained our results considering that in absence of $\beta-C D$, the azo dye is released from the aqueous core and the polar region of interphase. In the presence of $\beta-C D$, a part of the MO is included into the cavity, and in that way could be more available to the aqueous outside because the location of the macrocycle.

In the other hand, we mentioned that the lower affinity of MO for the cavities of Mod- $\beta$-CD in T80-S80 niosomes can be responsible of the lack of assistance of Mod- $\beta$-CD during the release experiments and only a release from the niosome was observed.

MY release from T80-S80 niosomes was slower than MO release and we explain these results considering that MY have a high affinity for niosomes due to its lower aqueous solubility, and the presence of Mod- $\beta$-CD do not alter the release profile as was expected considering the absence of interaction between MY and Mod- $\beta$-CD.

\section{Conclusions}

Spectrophotometric studies between molecular probes and cyclodextrin loaded niosomes enabled to infer about the location of the macrocyclic cavity and the dyes in the vesicular system.

The niosomes in presence of $\beta$-CD allowed to improve entrapped hydrophilic molecular probe amount and in turn, to produce a faster dye release. Even though $\beta$-CD presence does not modify considerably niosomes size and shape, positively modifies entrapment ability and release, particularly when the compound is hydrophilic and with certain affinity by cyclodextrin. The incorporation of Mod- $\beta-C D$, instead, did not produce entrapment and release differences.

The results presented here shows that cyclodextrin may form part of niosomes. The cyclodextrin modified niosomes have promising properties for entrapment and delivery of different molecules that may be interesting active compounds, fundamentally as food and pharmaceutical additives, due to the adequate nature of the components of the vesicles. We will continue exploring this interesting area.

\section{Conflicts of interest}

There are no conflicts of interest to declare.

\section{Acknowledgements}

This research was supported in part by Consejo Nacional de Investigaciones Científicas y Técnicas (CONICET, PIP 112201101-00441 y PIP 112-2015-01-00242), Agencia Nacional de Ciencia y Tecnología (FONCyT, PICT 2014-2516), Ministerio de Ciencia y Tecnología de la Provincia de Córdoba (MINCyT-Cba, PID 2009-2011) and Universidad Nacional de Córdoba, Argentina (SECyT 30720150100659CB). OFS, RHR and MAF hold researcher positions at CONICET. NDM thanks CONICET for the fellowship granted.

\section{Notes and references}

1 C. Y. Wong, H. Al-Salami and C. R. Dass, Int. J. Pharm., 2018, $537,223$.

2 M. Bai, J. He, L. Kang, J. Nie and R. Yin, Int. J. Biol. Macromol., 2018, 113, 889.

3 C. M. Asensio, A. J. Paredes, M. P. Martin, D. A. Allemandi, V. Nepote and N. R. Grosso, J. Food Sci., 2017, 82, 2864.

4 M. D. Chatzidaki, K. Papadimitriou, V. Alexandraki, F. Balkiza, M. Georgalaki, V. Papadimitriou, E. Tsakalidou and A. Xenakis, Food Chem., 2018, 255, 97.

5 S. Gopalakrishnan and A. Chenthilnathan, Res. J. Pharm., Biol. Chem. Sci., 2012, 3, 1090.

6 N. Akhtar, Curr. Drug Delivery, 2014, 11, 87.

7 M. Imran, M. R. Shah, F. Ullah, S. Ullah, A. M. A. Elhissi, W. Nawaz, F. Ahmad, A. Sadiq and I. Ali, Drug Delivery, 2016, 23, 3653.

8 E. Soo, S. Thakur, Z. Qu, S. Jambhrunkar, H. S. Parekh and A. Popat, J. Colloid Interface Sci., 2016, 462, 368.

9 Y. Liu, D. Liu, L. Zhu, Q. Gan and X. Le, Food Res. Int., 2015, 74, 97.

10 T. Uchino, F. Lefeber, G. Gooris and J. Bouwstra, Eur. J. Pharm. Biopharm., 2014, 86, 156.

11 L. Tavano and R. Muzzalupo, Colloids Surf., B, 2016, 147, 161. 12 D. J. McClements, Adv. Colloid Interface Sci., 2015, 219, 27.

13 C. Marianecci, L. Di Marzio, F. Rinaldi, C. Celia, D. Paolino, F. Alhaique, S. Esposito and M. Carafa, Adv. Colloid Interface Sci., 2014, 205, 187.

14 C. Marianecci, S. Petralito, F. Rinaldi, P. N. Hanieh and M. Carafa, J. Drug Delivery Sci. Technol., 2016, 32, 256.

15 P. Freixeiro, E. Diéguez-Casal, L. Costoya, B. Seijo, C. M. Ferreirós, M. T. Criado and S. Sánchez, Int. J. Pharm., 2013, 443, 1.

16 I. F. Uchegbu and A. T. Florence, Adv. Colloid Interface Sci., 1995, 58, 1.

17 I. F. Uchegbu and S. P. Vyas, Int. J. Pharm., 1998, 172, 33.

18 C. Marianecci, L. Di Marzio, E. Del Favero, L. Cantù, P. Brocca, V. Rondelli, F. Rinaldi, L. Dini, A. Serra, P. Decuzzi, C. Celia, D. Paolino, M. Fresta and M. Carafa, Langmuir, 2016, 32, 1241.

19 N. B. Mahale, P. D. Thakkar, R. G. Mali, D. R. Walunj and S. R. Chaudhari, Adv. Colloid Interface Sci., 2012, 183-184, 46.

20 D. Pando, G. Gutiérrez, J. Coca and C. Pazos, J. Food Eng., 2013, 117, 227.

21 S. Moghassemi and A. Hadjizadeh, J. Controlled Release, 2014, 185, 22.

22 M. Mashal, N. Attia, G. Puras, G. Martínez-Navarrete, E. Fernández and J. L. Pedraz, J. Controlled Release, 2017, 254, 55.

23 V. Sharma, S. Anandhakumar and M. Sasidharan, Mater. Sci. Eng., C, 2015, 56, 393.

24 S. K. Mehta and N. Jindal, Colloids Surf., B, 2013, 101, 434.

25 L. Tavano, R. Muzzalupo, N. Picci and B. De Cindio, Colloids Surf., B, 2014, 114, 144.

26 L. Tavano, R. Aiello, G. Ioele, N. Picci and R. Muzzalupo, Colloids Surf., B, 2014, 118, 7. 
27 D. Pando, M. Matos, G. Gutiérrez and C. Pazos, Colloids Surf., $B, 2015,128,398$.

28 A. H. Alomrani, M. H. Al-Agamy and M. M. Badran, J. Drug Delivery Sci. Technol., 2015, 28, 37.

29 D. Pando, M. Beltrán, I. Gerone, M. Matos and C. Pazos, Food Chem., 2015, 170, 281.

30 L. Zhang, Q. Zhang, X. Wang, W. Zhang, C. Lin, F. Chen, X. Yang and W. Pan, Int. J. Pharm., 2015, 492, 40.

31 C. Aloisio, S. G. Antimisiaris and M. R. Longhi, J. Mol. Liq., 2017, 229, 106.

32 R. Gharib, H. Greige-Gerges, S. Fourmentin, C. Charcosset and L. Auezova, Carbohydr. Polym., 2015, 129, 175.

33 E. M. M. del Valle, Process Biochem., 2004, 39, 1033.

34 O. Elsie, S. B. Tiwari, N. Udupa, K. Ravindra and D. Uma, Indian J. Pharmacol., 1999, 31, 279.

35 I. P. Sheena, U. V. Singh, A. Shirinivas K and N. Udupa, J. Pharm. Pharmacol., 1997, 3, 383.

36 The Merck Index. An Encyclopedia of Chemicals, Drugs, and Biologicals, ed. S. Budavari, Merck and Co., Inc., Whitehouse Station, NJ, 12th edn, 1996, p. 1041.

37 H. Jaber, W. Mabey, A. Liu, T. Chou, H. Johnson, T. Mill, R. T. Podoll and J. S. Winterle, Data Acquisition for Environmental Transport and Fate Screening for Compounds of Interest to the Office of Emergency and Remedial Response, EPA/600/6-84/011, U.S. Environmental Protection Agency, Washington, D.C., 1984, p. 43.

38 O. F. Silva, M. A. Fernández, S. L. Pennie, R. R. Gil and R. H. de Rossi, Langmuir, 2008, 24, 3718.

39 O. F. Silva, R. H. de Rossi, N. M Correa, J. J. Silber and R. D. Falcone, $R S C A d v .$, 2018, 8, 12535.

40 P. S. Sales, R. H. de Rossi and M. A. Fernández, Chemosphere, 2011, 84, 1700.

41 O. F. Silva, J. J. Silber, R. H. de Rossi, N. M. Correa and M. A. Fernández, J. Phys. Chem. B, 2007, 111, 10703.

42 O. F. Silva, N. M. Correa, J. J. Silber, R. H. de Rossi and M. A. Fernández, Langmuir, 2014, 30, 3354.
43 S.-F. Ng, J. J. Rouse, F. D. Sanderson, V. Meidan and G. M. Eccleston, AAPS PharmSciTech, 2010, 11, 1432.

44 M. Salim, H. Minamikawa, A. Sugimura and R. Hashim, Med. Chem. Commun., 2014, 5, 1602.

45 T. Yoshioka, B. Sternberg and A. T. Florence, Int. J. Pharm., 1994, 105, 1.

46 B. Maggio, Biochim. Biophys. Acta, Biomembr., 1985, 815, 245.

47 M. Rovira-Bru, D. H. Thompson and I. Szleifer, Biophys. J., 2002, 83, 2419.

48 A. H. Alomrani, G. A. Shazly, A. A. A. F. Amara and M. M. Badran, Colloids Surf., B, 2014, 121, 74.

49 M. Tsianou and A. I. Fajalia, Langmuir, 2014, 30, 13754.

50 J. J. Pinzón Barrantes, B. Maggio, R. H. De Rossi and R. V. Vico, J. Phys. Chem. B, 2017, 121, 4482.

51 D. D. Verma, S. Verma, G. Blume and A. Fahr, Int. J. Pharm., 2003, 258, 141.

52 L. Tavano, L. Gentile, C. Oliviero Rossi and R. Muzzalupo, Colloids Surf., B, 2013, 110, 281.

53 S. Allenmark, Chirality, 2003, 15, 409.

54 K. Harata and H. Uedaira, Bull. Chem. Soc. Jpn., 1975, 48, 375. 55 K. Harata, Bioorg. Chem., 1981, 10, 255.

56 J. Carrazana, B. Reija, P. R. Cabrer, W. Al-Soufi, M. Novo and J. Vázquez Tato, Supramol. Chem., 2004, 16, 549.

57 K. M. Tawarah and H. M. Abu-Shamleh, J. Inclusion Phenom. Mol. Recognit. Chem., 1991, 11, 29.

58 https://pubchem.ncbi.nlm.nih.gov.

59 Calculated using Chem Draw Ultra 8, Cambridge Soft. Corp., 1985-2003.

60 C. Zhou, X. Cheng, Q. Zhao, Y. Yan, J. Wang and J. Huang, Langmuir, 2013, 29, 13175.

61 S. S Dhule, P. Penfornis, T. Frazier, R. Walker, J. Feldman, G. Tan, J. He, A. Alb, V. John and R. Pochampally, Nanomedicine, 2012, 8, 440.

62 H. Chen, J. Gao, F. Wang and W. Liang, Drug Delivery, 2007, 14, 201. 